\title{
Incidencia y Características del Trauma Raquimedular en un Hospital de III Nivel, Bogotá 2011-2014
}

\author{
${ }^{1}$ Aníbal Alfonso Teherán, ${ }^{2}$ Oscar Javier Castro, ${ }^{3}$ Leonardo Laverde Frade
}

\section{RESUMEN}

Objetivo: La incidencia de Trauma Raquimedular (TRM) para Latinoamérica es estimada a partir de registros epidemiológicos de Brasil, por lo que decidimos medir la incidencia, distribución y características relacionadas con el TRM en una institución referente de III nivel.

Materiales y métodos: Se realizó un estudio descriptivoretrospectivo de pacientes atendidos durante 5 años en un Hospital de III nivel. Se estimó la incidencia mensual/anual de TRM (Io-TRM, IC95\%), se caracterizaron por variables sociodemográficas y clínicas, entre ellas: Condiciones al ingreso, clasificación de la lesión (AO, ASIA) y seguimiento funcional a 6 meses. Se realizó un análisis multivariado (ACM), para identificar variables relacionadas con mejoría funcional a los 6 meses.

Resultados: Se identificaron 174 casos, edad mediana 45 años, hombres $75,3 \% ; 70 \%$ se accidentó durante su actividad laboral, ninguno con un seguro de accidentes laborales. La lo-TRM del periodo fue 1,34 casos/100 personas-año (IC95\%;1,51-1,55). La estancia hospitalaria mediana fue 12 días, con diferencias al estratificar por la clasificación $A O$, ASIA-ingreso, tipo de manejo (médico/quirúrgico) y manejo en UCI (p: 0,000). La caída de altura y los accidente de tránsito, fueron la causa principal (88,5\%;IC95\%, 83,5-93,5\%); los traumas lumbares/torácicos se presentaron en $75,3 \%$ de los casos; el ASIA-ingreso fue D o E en $75 \%$ de los pacientes. Se identificó relación entre la mejoría a 6 meses y las categorías $D$ y C, según las clasificaciones ASIA y AO, respectivamente.

Conclusión: La lo-TRM del HUS fue relativamente mayor que la de otras series locales, la EH se relacionó con el tipo/lugar de tratamiento y severidad de la lesión, esta última determinó la mejoría a los 6 meses.

Importancia clínica: Se requiere crear un sistema de vigilancia, ajustado a modelos internacionales para medir el impacto real de esta patología.

\footnotetext{
${ }^{1}$ Residente de Medicina de Emergencias, Universidad del Rosario, ${ }^{2,3}$ Neurocirujano

${ }^{1}$ Centro de Investigación, Fundación Universitaria Juan N Corpas, Bogotá DC, Colombia

${ }^{2}$ Neurocirugía, Fundación Valle del Lili, Cali Colombia

${ }^{3}$ Neurocirugía, Hospital Universitario La Samaritana, Bogotá DC, Colombia

Corresponding Author: Aníbal Alfonso Teherán, Residente de Medicina de Emergencias, Centro de Investigación, Fundación Universitaria Juan N Corpas, Bogotá DC, Colombia, Phone: +8014961, e-mail: anibal.teheran@juanncorpas.edu.co
}

Palabras claves: Clasificación AO, Clasificación ASIA, Incidencia, Trauma raquimedular.

How to cite this article: Teherán AA, Castro OJ, Frade LL. Incidencia y Características del Trauma Raquimedular en un Hospital de III Nivel, Bogotá 2011-2014. Panam J Trauma Crit Care Emerg Surg 2016;5(3):140-147.

Source of support: Nil

Conflict of interest: None

\section{ABSTRACT}

Introduction: The incidence of Spinal Cord Injury (SCI) for Latin America has been estimated from epidemiological researches of Brazil, so we decided to measure the incidence, distribution and features related to $\mathrm{SCl}$ in a reference level 3 institution.

Materials and methods: A descriptive retrospective study was done with patients attended during 5 years at a level 3 hospital. Monthly and annual SCI (loSCl, Cl95\%) incidence was estimated; they were categorized by sociodemographic and clinical variables, among these: Conditions at hospital admission, severity of injury classification (AO, ASIA) and 6-month functional follow-up; a multiple correspondence analysis (MCA) was done, to extract component related with functional improvement at 6 months.

Results: A total of 174 cases were identified, median age of 45 years, men $75.3 \% ; 70 \%$ were injured during work activity, none of that with labor accident insurance. The lo-SCI from the period was 1.34 cases/100 person-year (CI95\%, 1.51-1.55). The average in-hospital length of stay was 12 days (LOS), with differences among AO classification, ASIA-admission, type of management (medical/surgical) and admission to ICU (p:0.000). Falls and motor vehicle crashes, both were the main cause (88.5\%, 95\% $\mathrm{Cl}, 83.5-93.5 \%$ ); lumbar and thoracic injuries, both were present in $75.3 \%$ of the cases; admission ASIA was D or E in $75 \%$ of the patients. Functional improvement at 6 -months was present when, at admission, ASIA classification it was D and AO was $C$.

Conclusion: The lo-SCI was higher than other local series, the LOS was related with the type, place of treatment and severity of the injury; in turn, severity of injury was related with functional improvement at 6 months.

Clinic relevance: Colombia needs to create a surveillance system adjusted to international standard, to evaluate the impact of this condition.

Keywords: Spinal cord injury, incidence, ASIA classification, AO classification.

\section{INTRODUCCIÓN}

Se denomina trauma raquimedular (TRM) a cualquier lesión que comprometa la columna vertebral, la medula 
espinal o sus estructuras de sostén. Es una patología muy antigua, que desde el 2000 antes de Cristo ha representado un reto para la comunidad médica en general y que actualmente presenta insuficientes datos epidemiológicos.

La incidencia global es cercana a 23 casos por cada millón de personas ${ }^{1}$ y la información disponible sólo permite hacer comparaciones entre algunas regiones, Norteamérica presenta 40 casos/millón, el oeste de Europa 15 casos/millón y Australia 16 casos/millón. Particularmente América Latina tiene muy pocos datos, que provienen principalmente de Sao Paulo y se extrapolan al resto del continente. ${ }^{1}$ En Estados Unidos (EUA) el TRM se presenta en 1 de cada 40 pacientes que ingresan a urgencias por algún tipo de trauma. ${ }^{1}$ La mortalidad en el sitio del evento o en las salas de urgencias puede llegar hasta $48,3-79 \%$ de los pacientes. $^{2}$

El TRM es un evento traumático que frecuentemente culmina en dependencia absoluta o parcial de la sociedad, finalización de la vida laboral o cambio a una actividad diferente a la profesión del lesionado y altos costos por atención médica o soportes de cuidado, aún 6 años después de la ocurrencia del evento. ${ }^{3}$ Los pacientes con TRM generan un costo promedio, en dólares, de \$35.000 por hospitalización y su estancia hospitalaria es 2,5 veces la presentada por pacientes que sólo requirieron manejo ambulatorio. ${ }^{4}$ Durante el primer año de hospitalización el costo de los pacientes con tetraplejia alta fue mayor al $\$ 1.000 .000$, con tetraplejia baja $\$ 754.000$, con paraplejia $\$ 500.000$ y con lesión motora incompleta/funcional en cualquier nivel $\$ 340.000 .^{5}$

Dado los costos de este evento, fue necesario indagar en metodologías para estimar la incidencia. ${ }^{6} \mathrm{La}$ Organización Mundial de la Salud (OMS), implementó un modelo de regresión, que utilizó la información contenida en base de datos diseñadas para la vigilancia prospectiva, registros de bases de datos administrativas e información contenida en manuscritos científicos publicados hasta el año 2011. ${ }^{6}$ Brasil fue el único país de Latinoamérica que presentaba información útil para estimar la incidencia del TRM, por lo tanto, se convirtió en nuestro referente de comparación. Una revisión sistemática de la literatura determinó la carencia de estudios en Latinoamérica y la necesidad de realizar investigaciones que permitan estimar el verdadero riesgo de nuestra población. ${ }^{7}$

Después de realizar una búsqueda sistemática específica (Material Suplementario), dirigida a identificar manuscritos orientados a describir la frecuencia/epidemiología del TRM en Colombia, hasta noviembre/2015 en las bases de datos PubMed y BVS/BIREME, sólo encontramos una investigación, que después de 5 años de revisión retrospectiva de registros médicos, identificó una frecuencia de 12 casos por cada 1000 pacientes que ingresaron a la Unidad de Cuidados Intensivos (UCI) de una institución de III nivel. ${ }^{8}$
En Colombia, el hospital Universitario de La Samaritana (HUS) es un referente de atención para pacientes con TRM, por lo que decidimos medir la incidencia, distribución y características relacionadas con el TRM en pacientes atendidos en el Servicio de Neurocirugía durante 5 años, con el fin de aportar información que permita tomar decisiones en materia de Salud Pública para el manejo y seguimiento de esta patología.

\section{MATERIALES Y MÉTODOS}

\section{Diseño, lugar y selección de pacientes}

Se realizó un estudio descriptivo retrospectivo, utilizando los registros médicos de pacientes atendidos por TRM en el Servicio de Neurocirugía, del HUS, durante el periodo 2010-2014. Se seleccionaron todos los pacientes diagnosticados con TRM durante el periodo mencionado y se excluyeron los pacientes con más de 15 días de evolución de trauma, con manejo quirúrgico inicial en otra institución o con procesos infecciosos previos (Figura S1).

Los análisis y reportes de esta investigación fueron realizados basados en la guía metodológica Strenghtening the Reporting of Observational Studies in Epidemiology (STROBE). ${ }^{9}$

\section{Descripción de la base de datos}

Se incluyeron variables sociodemográficas y clínicas sobre las condiciones de ingreso del paciente, clasificación de la lesión y seguimiento del paciente hasta 6 meses posteriores al ingreso, a partir de la información contenida en los registros médicos.

\section{Variables}

Se estimó la incidencia (Io, IC95\%) tipo proporción de TRM, a partir del número de casos (anuales o mensuales), sobre el número de pacientes atendidos en el Servicio de Neurocirugía, en un periodo de tiempo específico, por cada 100 pacientes atendidos.

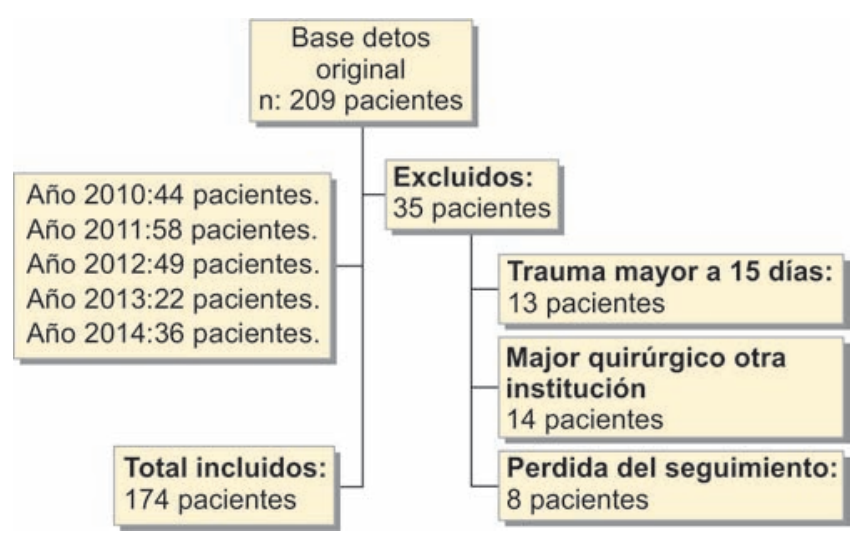

Figura S1: Flujograma de selección de pacientes 
Se describió la distribución mensual de casos y se estimó (IC95\%) el promedio de eventos mensuales en cada uno de los años estudiados.

Se evaluó la distribución de casos por sexo, edad, estado civil, ocupación, régimen de atención, momento de la semana de ingreso y lugar del accidente.

Se determinó la mediana (p25-p75) de estancia hospitalaria $(\mathrm{EH})$ y su relación con variables sociodemográficas o clínicas; se recolectó información sobre el mecanismo del trauma, nivel espinal de la lesión, estado funcional al ingreso con la escala de Karnofsky, ${ }_{10}^{10}$ puntaje de comorbilidad según la escala de Charlson, ${ }^{11}$ tipo de tratamiento (médico/quirúrgico), manejo quirúrgico por neurocirugía $\mathrm{u}$ otra especialidad adicional, necesidad de manejo en UCI, uso de esteroides, necesidad de rehospitalización, resultado positivo para alcoholemia, clasificación de la lesión según la clasificación $\mathrm{AO}$ al ingreso ${ }^{12}$ y según el estándar American Spinal Injury Association (ASIA) al ingreso, al momento del alta hospitalaria y a los 6 meses de seguimiento. ${ }^{13}$

\section{Análisis estadísticos}

Se utilizaron los paquetes estadísticos Minitab versión 17 y Epidat versión 3.1 para analizar los datos. Los datos se expresaron en medianas (p25-p75) o medias (IC95\%) y proporciones; se determinó normalidad con la prueba de Shapiro Wilks; las comparaciones entre datos continuos, para dos grupos independientes, se realizaron con una prueba T de Student o U Mann Whitney en caso de distribuciones asimétricas; para 3 o más grupos independientes, con un ANOVA o Kruskall Wallis en caso de distribuciones asimétricas; los datos categóricos se compararon con la prueba de Chi cuadrado $\left(\chi^{2}\right)$; se estableció una $\mathrm{P}<0,05$ (dos colas) como significativa.

Los pacientes se reclasificaron en aquellos con o sin mejoría a los 6 meses según la clasificación ASIA y se realizó un análisis de correspondencias múltiples (ACM), con las variables que preliminarmente presentaran asociación estadística $\left(\chi^{2} ; \mathrm{P}:<0,05\right)$, con el fin de corroborar las asociaciones identificadas.

\section{Aspectos éticos}

Esta investigación se clasificó sin riesgo, según la Resolución 008430/1993; se ejecutó después de la aprobación del Comité de Ética y de Investigaciones del HUS, y el Centro de Investigación de la Fundación Universitaria Juan N. Corpas.

\section{RESULTADOS}

Se identificaron 174 casos de TRM, con edad entre 15-83 años y en todos los años, exceptuando el 2013, la mayoría eran hombres (IC95\%, 68,9-81,7\%) y estaban casados o en unión libre (IC95\%, 95,5-99,5\%) (Tabla 1, Gráfico S1). Más del $70 \%$ de los casos trabajaban en labores de explotación, transporte y oficios varios y ninguno estaba afiliado a una Administradora de Riesgos Laborales (ARL); los casos provenían predominantemente de Cundinamarca (IC95\%, 69,5-82,2\%) e ingresaron con mayor frecuencia los días sábados (18,4\%; IC95\%, $12,6-24,1 \%)$.

Tabla 1: Características sociodemográficas de pacientes atendidos con TRM en el Hospital la Samaritana, desde enero 2011-enero 2015

\begin{tabular}{|c|c|}
\hline Características generales & $n: 174$ \\
\hline Edad, mediana (Q1-Q3) & $12(8-20)$ \\
\hline Hombres, n (\%) & $131(75,3)$ \\
\hline \multicolumn{2}{|l|}{ Estado civil, $n(\%)$} \\
\hline Casado-Unión libre & $89(51,1)$ \\
\hline Soltero & $79(45,4)$ \\
\hline Separado-Viudo & $6(3,5)$ \\
\hline \multicolumn{2}{|l|}{ Ocupación, n (\%) } \\
\hline Explotación primaria y extractiva & $82(47,1)$ \\
\hline Transporte y oficios varios & $48(27,6)$ \\
\hline Ventas y servicios & $17(9,8)$ \\
\hline Otros & $27(15,5)$ \\
\hline \multicolumn{2}{|l|}{ Régimen de atención, $n$ (\%) } \\
\hline Subsidiado & $102(58,6)$ \\
\hline SOAT & $52(29,9)$ \\
\hline Contributivo & $20(11,5)$ \\
\hline \multicolumn{2}{|l|}{ Momento de ingreso, $n(\%)$} \\
\hline Lunes-jueves & $101(58,0)$ \\
\hline Viernes-domingo & $73(41,9)$ \\
\hline \multicolumn{2}{|l|}{ Lugar del accidente, $n(\%)$} \\
\hline Cundinamarca & $132(75,9)$ \\
\hline Bogotá & $30(17,2)$ \\
\hline Otro departamento & $12(6,9)$ \\
\hline
\end{tabular}

Ocupación: Según clasificación nacional de ocupaciones SENA

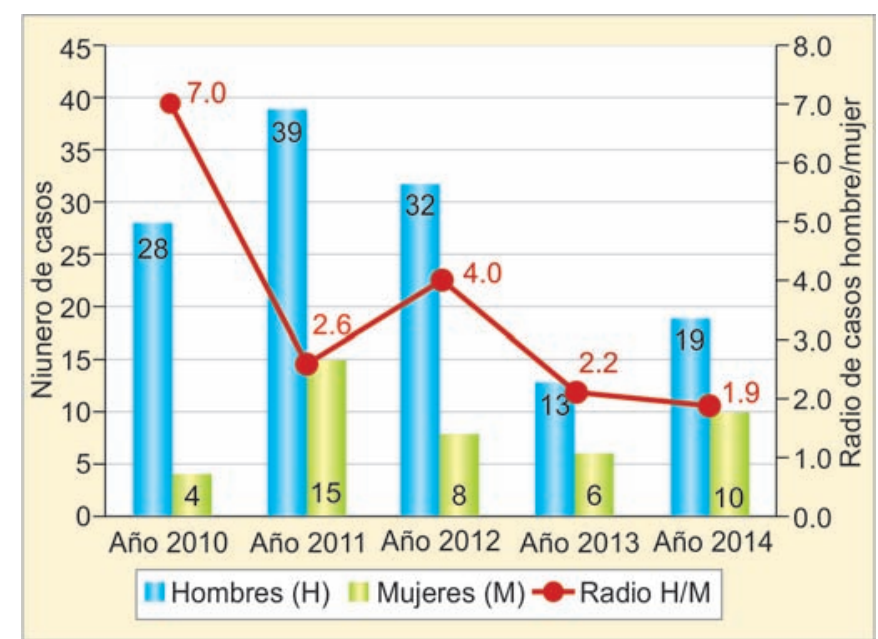

Gráfico S1: Comparación de frecuencia de casos de TRM, según sexo y año de presentación, de pacientes atendidos en el Servicio de Neurocirugía, HUS, 2010-2014; TRM: Trauma raquimedular; HUS: Hospital Universitario La Samaritana. ${ }^{* * *} \mathrm{p}:<0,001,{ }^{*} \mathrm{p}:<0,05$ 


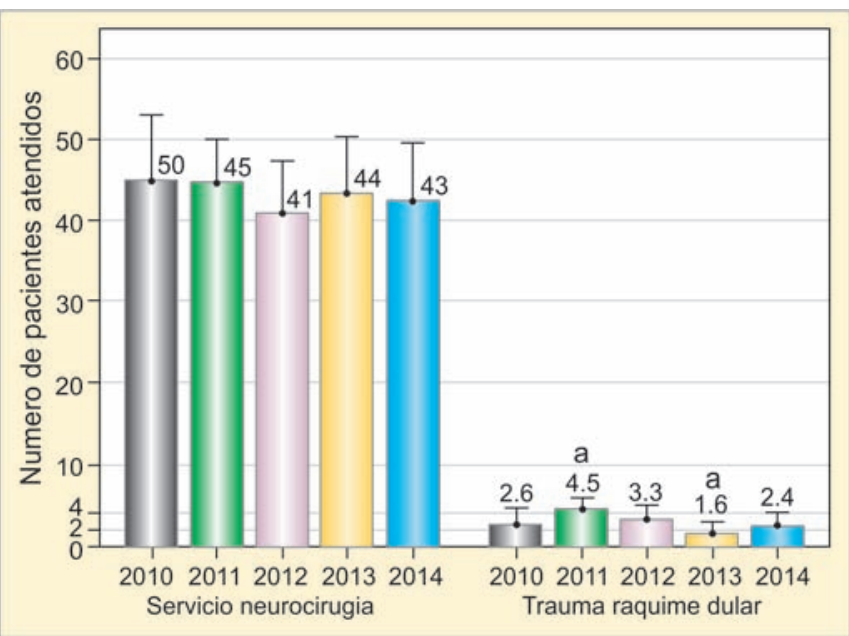

Gráfico 1: Promedio mensual de pacientes atendidos en el Servicio de Neurocirugía y de pacientes con TRM, HUS, 2010-2014; El número de casos mensuales se utilizó para estimar el promedio (IC95\%) de casos anuales, de pacientes atendidos en el Servicio de Neurocirugía o que ingresaron por TRM en el Hospital Universitario La Samaritana (HUS). Las comparaciones se realizaron con una prueba ANOVA a 1 vía; a: $p<0,001$

\section{Incidencia de Trauma Raquimedular (Io-TRM)}

El Servicio de Neurocirugía atendió mensualmente una media (DE) de $43 \pm 7$ casos, $3 \pm 2$ correspondieron a casos de TRM; no se encontraron diferencias en el promedio de pacientes mensuales atendidos por el Servicio de Neurocirugía entre los años estudiados, pero si en el promedio mensual de casos de TRM por año, el 2011 presentó el mayor promedio de casos mensuales y el 2013 el menor promedio de casos mensuales ( $\mathrm{p}:<0,05)$ (Gráfico 1); la totalidad de casos se distribuyó en meses con frecuencias $\geq 10 \%$ (enero, febrero, abril, octubre), entre 6-8\% (mayo, noviembre, diciembre, marzo) $y \leq 5 \%$ (agosto, septiembre, julio) (Gráfico S2).

La Io-TRM (personas-año) durante el periodo 20102014 fue de 1,34 casos por cada 100 personas año $(1,51-1,55)$; durante el año 2012 y 2011 se presentaron la mayor y menor Io-TRM, respectivamente (Tabla 2).

En los meses de enero y abril se identificaron las Io-TRM más altas y en julio la más baja (Tabla S1, Material Suplementario).

Tabla 2: Incidencia de TRM anual, en pacientes atendidos en el Servicio de Neurocirugia del Hospital La Samaritana, 2010-2014

\begin{tabular}{lllll}
\hline Periodo & Neurocirugía (n) & TRM (n) & $\begin{array}{l}\text { lo (Tipo } \\
\text { proporción) }\end{array}$ & IC95\% \\
\hline Año 2010 & 539 & 32 & 5,94 & $3,85-8,03$ \\
Año 2011 & 537 & 54 & 10,05 & $7,42-12,69$ \\
Año 2012 & 489 & 40 & 8,18 & $5,65-10,71$ \\
Año 2013 & 523 & 19 & 3,63 & $1,93-5,33$ \\
Año 2014 & 510 & 29 & 5,69 & $3,57-7,79$ \\
\hline
\end{tabular}

TRM: Trauma raquimedular. Io: Incidencia

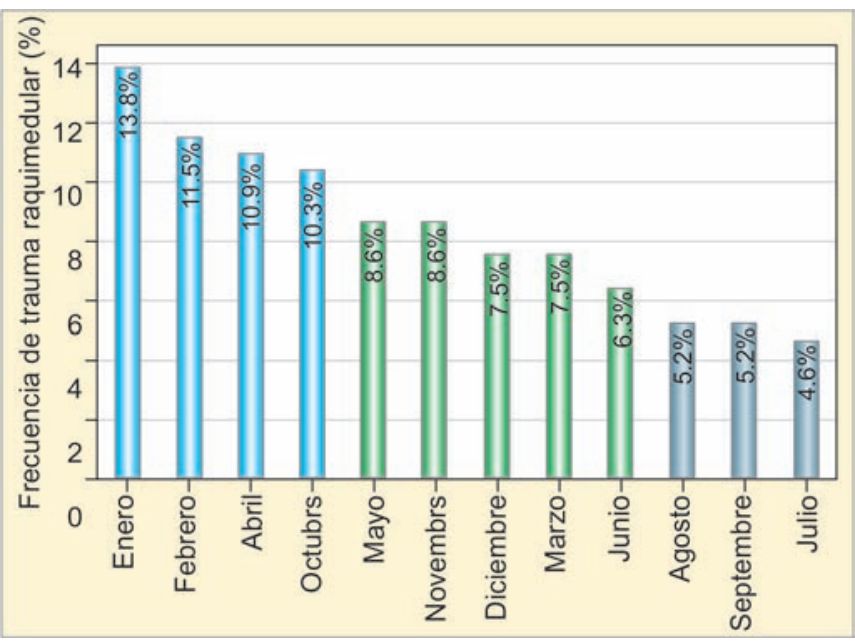

Gráfico S2: Distribución de casos de Trauma Raquimedular por mes, durante el periodo 2010-2014, Hospital Universitario La Samaritana Se calculó a partir de la división del número de casos de trauma raquimedular por mes sobre el total de casos atendidos durante el periodo de estudio

\section{Características clínicas de pacientes con TRM}

La EH (mediana) fue 12 días, sin diferencias entre los años estudiados, pero con diferencias al evaluar según las clasificaciones $\mathrm{AO}$, ASIA al ingreso, tipo de manejo (médico/quirúrgico) y necesidad de manejo en UCI.

Según la clasificación AO, los pacientes con categoría A presentaron menor EH que aquellos en categoría B o C; según la clasificación ASIA al ingreso, los pacientes con categoría A presentaron mayor EH que aquellos en categoría D o E y los pacientes con categoría C presentaron mayor EH que aquellos en categoría E (Gráfico 2A y 2B). En comparación con la estancia hospitalaria general, los pacientes que recibieron manejo médico presentaron

Tabla S1: Incidencia de trauma raquimedular por por mes, en pacientes atendidos en el Servicio de Neurocirugia del Hospital La Samaritana, 2010-2014

\begin{tabular}{lllll}
\hline Periodo & $\begin{array}{l}\text { Neurocirugía } \\
(n)\end{array}$ & $\begin{array}{l}\text { TRM } \\
(n)\end{array}$ & $\begin{array}{l}\text { lo (Tipo } \\
\text { proporción) }\end{array}$ & IC95\% \\
\hline Enero & 200 & 24 & 12,0 & $7,24-16,75$ \\
Febrero & 169 & 20 & 11,83 & $6,66-17,0$ \\
Marzo & 163 & 13 & 7,97 & $3,51-12,44$ \\
Abril & 147 & 19 & 12,92 & $7,16-18-68$ \\
Mayo & 176 & 15 & 8,52 & $4,11-12,93$ \\
Junio & 160 & 11 & 6,87 & $2,64-11,10$ \\
Julio & 191 & 8 & 4,18 & $1,08-7,29$ \\
Agosto & 164 & 9 & 5,48 & $1,69-9,27$ \\
Septiembre & 183 & 9 & 4,91 & $1,51-8,32$ \\
Octubre & 201 & 18 & 8,95 & $4,75-13,5$ \\
Noviembre & 156 & 15 & 9,61 & $4,66-14,56$ \\
Diciembre & 195 & 13 & 6,66 & $2,90-10,42$ \\
\hline
\end{tabular}

TRM: Trauma raquimedular; lo: Incidencia 


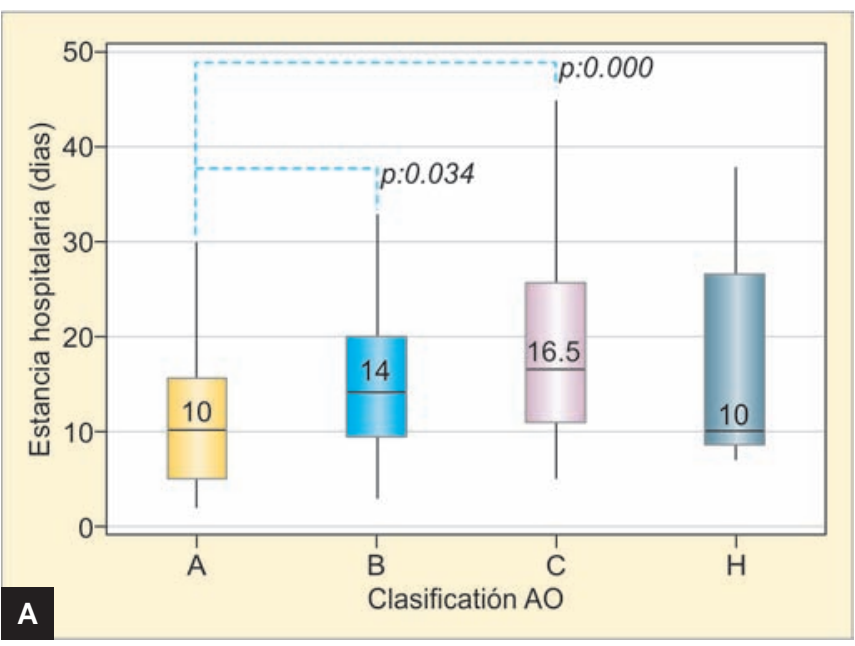

Gráfico 2A: Estancia hospitalaria según clasificación $A O$; Se utilizó la prueba $U$ Mann Whitney para comparar entre las parejas de categorías AO. TRM: Trauma raquimedular; HUS: Hospital Universitario La Samaritana

menor $\mathrm{EH}$ y los pacientes con manejo quirúrgico, tratados en UCI o por Neurocirugía en conjunto con otra especialidad, presentaron mayor EH; además, los pacientes que recibieron manejo médico, no manejados por otra especialidad quirúrgica y no manejados en UCI, presentaron menores $\mathrm{EH}$, en relación con sus respectivas contrapartes (p: <0,000) (Gráfico 3).

Las causas del trauma en $8 / 10$ casos fueron caída de altura o accidente de tránsito (88,5\%; IC95\%, 83,5-93,5\%); cerca de la mitad de los pacientes presentó trauma a nivel lumbar y la mayoría sólo requirió manejo médico, no ameritó manejo en UCI, uso de esteroides o re-hospitalización (Gráfico S3, Tabla 3).

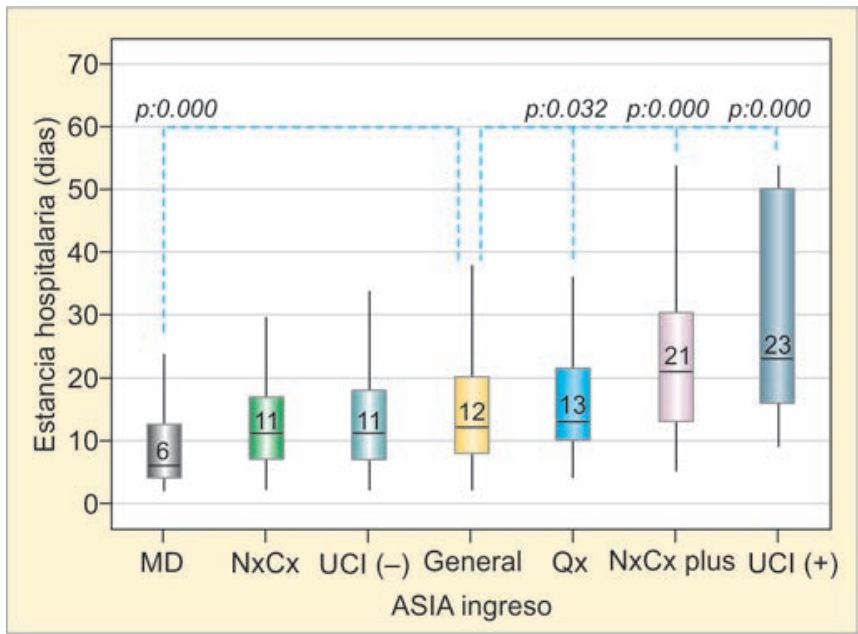

Gráfico 3: Comparación entre estancia hospitalaria general de pacientes con TRM y de pacientes con intervenciones específicas; Se utilizó la prueba U Mann Whitney para comparar entre los subgrupos. TRM: Trauma raquimedular; HUS: Hospital Universitario La Samaritana; EH: Estancia hospitalaria; MD: Médico; Qx: Quirúrgico; NxCx: Neurocirugía; NxCx_Plus: Neurocirugía y otra especialidad; UCI (-): No requirió ingreso a Unidad de Cuidados Intensivos. $\mathrm{UCl}(+)$ : Requirió ingreso a Unidad de Cuidados Intensivos

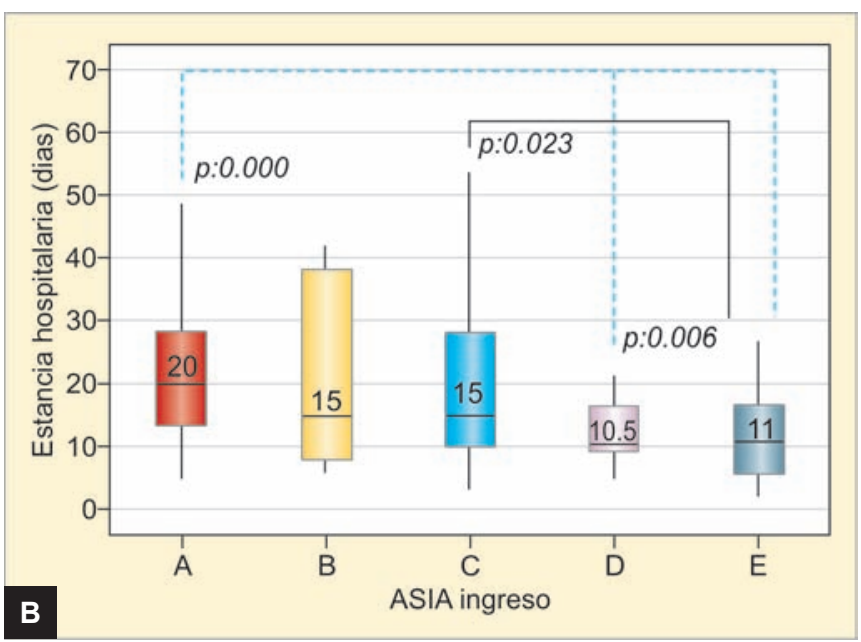

Gráfico 2B: Estancia hospitalaria según clasificación ASIA; Se utilizó la prueba U Mann Whitney para comparar entre las parejas de categorías ASIA. TRM: Trauma raquimedular; HUS: Hospital Universitario La Samaritana

\section{Clasificación de severidad y dis-habilidad al ingreso}

$\mathrm{Al}$ ingreso, 3/4 pacientes fueron clasificados en las categorías de ASIA D o E y cerca del 15\% con clasificación A; durante los 6 meses de seguimiento no se evidenció mejoría en la clasificación de ASIA para estos últimos.

La clasificación ASIA al ingreso, al momento del alta, la clasificación AO y el tipo de manejo (médico/quirúrgico), se relacionaron con cambios en la clasificación de ASIA a los 6 meses (Tabla 4). El ACM ejecutado entre las variables: Mejoría a los 6 meses (sí/no), ASIA al ingreso (A-B-CD-E), ASIA al alta de la hospitalización (A-B-C-D-E), clasificación $\mathrm{AO}(\mathrm{A}-\mathrm{B}-\mathrm{C}-\mathrm{H})$ y tipo de tratamiento (médico/ quirúrgico), identificó relación entre los pacientes con mejoría a los 6 meses y los categorizados en $\mathrm{D}$, según la clasificación ASIA al momento del ingreso, y los categorizados en $\mathrm{C}$ según la clasificación $\mathrm{AO}$ (Gráfico $\mathrm{S4}$ ).

\section{DISCUSIÓN}

Durante un quinquenio de estudio, de manera retrospectiva determinamos la incidencia de TRM en un centro de referencia y describimos características relacionadas con la clasificación de la lesión al ingreso y a 6 meses de seguimiento.

Nuestros hallazgos pueden deberse a las características sociodemográficas de los pacientes que ingresan al HUS, provenientes principalmente de municipios cercanos al Distrito Capital, ubicados en áreas rurales o en proceso de urbanización, donde las actividades laborales predominantes son: Agricultura, manejo de cargas pesadas, transporte, minería y otros dependientes de la vida campesina. En nuestro contexto social, frecuentemente estas actividades se ejecutan en el marco 


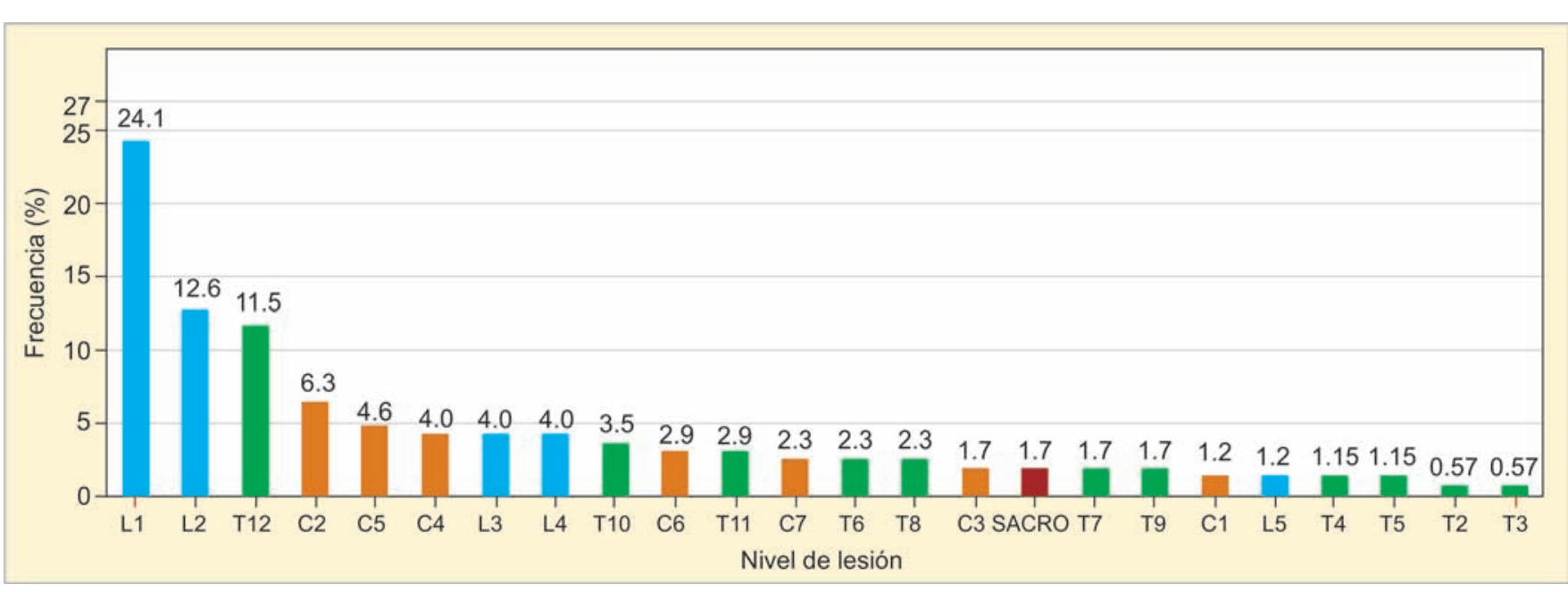

Gráfico S3: Distribución de casos de TRM según nivel de lesión, en pacientes con TRM, atendidos en el Servicio de Neurocirugía, HUS, 2010-2014; La frecuencia de casos por nivel de lesión, se calculó sobre el total de casos. C1-C7: Cervical (barras sin relleno), T1-T12: Torácica (barras grises), L1-L5: Lumbar (barras negras), Sacro: barras con líneas diagonales; TRM: Trauma raquimedular; HUS: Hospital Universitario La Samaritana

de un empleo informal, razón que explicaría la falta de afiliación laboral.

El perfil sociodemográfico de los pacientes que sufrieron TRM fue de hombres, adultos-jóvenes, con pareja estable y provenientes de la región de Cundinamarca; las 3 primeras características encierran el perfil del trabajador rural colombiano, que son consistentes con lo identificado en dos estudios realizados en ciudades capitales, pero que no detallaron el lugar de procedencia de los pacientes; ${ }^{8,14}$ asimismo, una RSL que estudió la epidemiología del

Tabla 3: Características clínicas de pacientes atendidos con TRM en el Hospital la Samaritana, desde enero 2011-enero 2015

\begin{tabular}{ll}
\hline Características clínicas & $n: 174$ \\
\hline Estancia hospitalaria, mediana (Q1-Q3) & $12(8-20)$ \\
Mecanismo de lesión, $n$ (\%) & \\
$\quad$ Caída & $105(60,3)$ \\
Accidente de tránsito & $49(28,2)$ \\
Trauma contuso & $11(6,3)$ \\
Lesión por agresión & $9(5,2)$ \\
Nivel de lesión, $n$ \% & \\
Cervical & $40(23,0)$ \\
Torácica & $51(29,3)$ \\
Lumbar & $80(46,0)$ \\
Sacra & $3(1,7)$ \\
Karnofski, $n$ (\%) & \\
100 & $170(97,7)$ \\
90-80 & $4(2,3)$ \\
Tratamiento médico, $\mathrm{n}(\%)$ & $125(72,8)$ \\
Manejo en UCl, $\mathrm{n}(\%)$ & $11(6,3)$ \\
Uso de esteroides, $\mathrm{n}(\%)$ & $6(3,5)$ \\
Detección de alcohol etílico, $n$ (\%) & \\
No & $150(86,2)$ \\
Sí & $15(8,6)$ \\
Indeterminado & $9(5,2)$ \\
Re-hospitalización, $n$ (\%) & $9(5,2)$ \\
\hline Trauma contuso: Trauma directo sobre colum
\end{tabular}

Trauma contuso: Trauma directo sobre columna vertebral secundario a impacto con objeto pesado
TRM de países en vías de desarrollo, identificó mayor frecuencia de TRM en hombres, adultos-jóvenes, ${ }^{7}$ esta incluyó dos estudios con población rural, llevados a cabo en Nepal y Turquía y ambos encontraron características demográficas similares al nuestro. ${ }^{15,16}$

En nuestra investigación la Io-TRM se estimó por cada 100 personas-año atendidas en el Servicio de Neurocirugía y a diferencia de lo encontrado en el estudio de Carvajal y colaboradores, que reporto la frecuencia de TRM por cada 1000 personas, este evento fue más

Tabla 4: Clasificaciones de las lesiones y dis-habilidad al ingreso del TRM y desenlace a los 6 meses de seguimiento

\begin{tabular}{|c|c|c|c|c|}
\hline \multirow[b]{2}{*}{ Clasificación } & \multicolumn{3}{|c|}{ Desenlace positivo a los 6 meses (ASIA) } & \multirow[b]{2}{*}{ Valor-P } \\
\hline & Sín (\%) & No $n(\%)$ & Total $n=174$ & \\
\hline \multicolumn{5}{|l|}{ ASIA ingreso } \\
\hline A & - & $25(16,0)$ & $25(14,3)$ & \\
\hline B & - & $5(3,2)$ & $5(2,9)$ & \\
\hline C & $11(61,1)$ & $13(8,3)$ & $24(13,8)$ & 0,000 \\
\hline $\mathrm{D}$ & $7(38,9)$ & $5(3,2)$ & $12(6,9)$ & \\
\hline$E$ & - & $108(69,2)$ & $108(62,1)$ & \\
\hline \multicolumn{5}{|l|}{ ASIA alta } \\
\hline A & - & $25(16,0)$ & $25(14,3)$ & \\
\hline B & - & $4(2,6)$ & $4(2,3)$ & \\
\hline $\mathrm{C}$ & $8(55,6)$ & $14(8,9)$ & $22(12,6)$ & 0,000 \\
\hline $\mathrm{D}$ & $10(44,4)$ & $6(3,8)$ & $16(9,2)$ & \\
\hline$E$ & - & $107(68,6)$ & $107(61,5)$ & \\
\hline \multicolumn{5}{|c|}{ Clasificación AO } \\
\hline A & $3(16,6)$ & $81(51,9)$ & $84(48,2)$ & \\
\hline B & $5(27,8)$ & $32(20,5)$ & $37(21,3)$ & \\
\hline C & $9(50)$ & $39(25,0)$ & $48(27,6)$ & 0,033 \\
\hline $\mathrm{H}$ & $1(5,6)$ & $4(2,6)$ & $5(2,9)$ & \\
\hline \multicolumn{5}{|l|}{ Karnofsky } \\
\hline 100 & $1(5,6)$ & $3(1,9)$ & $4(2,3)$ & 0,330 \\
\hline 90-80 & $17(94,4)$ & $153(98,1)$ & $170(97,7)$ & \\
\hline
\end{tabular}

Se implementó la prueba de independencia Chi cuadrado 


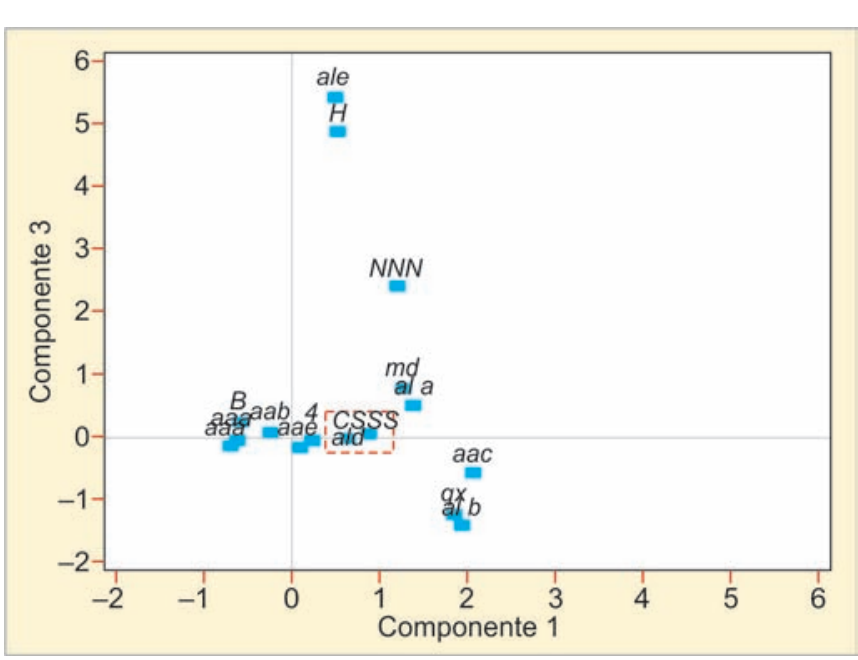

Gráfico S4: Gráfico de columnas entre clasificación de ASIA al ingreso, al alta, a los 6 meses clasificación AO y tipo de tratamiento; SSS: Sí presentó mejoría a los 6 meses; NNN: No presentó mejoría a los 6 meses; Clasificación ASIA al ingreso: Ala, alb, alc, ald, ale; Clasificación AO: A, B, C, H; ASIA al alta: aaa, aab, aac, aad, aae; Manejo médico: Md; Manejo quirúrgico: Qx

frecuente en nuestra institución. ${ }^{8}$ En ambos estudios no se obtuvieron los referentes poblacionales, motivo que no permitió compararnos con incidencias internacionales expresadas en casos por cada millón de habitantes. ${ }^{1,6,7}$

La mediana de EH evidenció una distribución asimétrica, comportamiento relacionado con la severidad de la lesión al ingreso, el tipo de manejo establecido y la necesidad de manejo en UCI. Dos estudios realizados a nivel local y otro internacional, evidenciaron una mediana (25-75) de EH 17 días (9-30) y una media (DE) de 12,4 (0,51) días;, ${ }^{4,7}$ el realizado a nivel local evidenció mayor EH que el nuestro, posiblemente porque éste sólo incluyó pacientes de UCI, variable que relacionamos con mayor EH; cabe mencionar que también evidenciaron variabilidad en la EH al estratificar según el nivel de la lesión, hallazgo contrario a nuestros resultados y donde no evidenciamos diferencias estadísticas.

Las causas más frecuentes de TRM en el HUS fueron la caída de altura, seguido de los accidentes de tránsito, estudios previos han evidenciado dos perfiles causales, en países desarrollados la causa más frecuente se relaciona con caídas de altura y en países en vías de desarrollo es el accidente de tránsito; $7,17,18$ nuestros resultados pueden deberse a que la explotación primaria y extractiva es el tipo de labor desempeñada más frecuentemente desempeñada, y esta a su vez es una causa frecuente de TRM en países en vías de desarrollo. ${ }^{7}$

Aproximadamente la mitad de pacientes presentaron compromiso a nivel lumbar, constituyendo la principal lesión encontrada, contrario a lo identificado en la mayoría de estudios de ámbito local e internacional, donde el nivel cervical ocupó la primera posición. ${ }^{8,19}$ Aunque no encontramos asociación estadística entre el nivel lumbar y las caídas de altura, éste patrón fue consistente con otras series donde el nivel lumbar fue el más frecuente. ${ }^{20-22}$

La severidad de la lesión y el manejo establecido al ingreso se relacionaron con mejoría a los 6 meses, cabe aclarar que la severidad de la lesión direcciona el manejo inicial del TRM, vínculo identificado en nuestro estudio (dato no mostrado). Estos resultados fueron consistentes con hallazgos previos que identificaron a la clasificación ASIA al ingreso, como un factor relacionado con el desenlace a los 6 meses o al año. ${ }^{14,23}$

\section{LIMITACIONES}

Entre las limitaciones que presentamos están la imposibilidad de compararnos con otras incidencias mundiales, por la no disponibilidad de denominadores poblacionales; además, no incluimos pacientes con TRM tratados por otras especialidades y aquellos no hospitalizados en el Servicio de Neurocirugía, información que pudo afectar la Io-TRM; por último, no caracterizamos los pacientes según la limitación funcional definitiva y desenlaces de importancia clínica como la aparición de eventos trombóticos, infecciosos y la necesidad de ventilación mecánica.

Con estos resultados concluimos que la Io-TRM del HUS fue relativamente mayor que la reportada en otras series locales y la duración de EH es dependiente de la severidad de lesión, del manejo instaurado y la necesidad de ingreso a UCI; los pacientes se caracterizaron por presentar lesiones lumbares/torácicas, presentando una caída de altura con principal etiología y su mejoría a los 6 meses se relacionó con la severidad de la lesión.

Dada la falta de información estadística sobre el TRM en Colombia y el mundo, esto nos abre una puerta para integrarnos a sistemas de vigilancia y control de otros países con problemáticas similares al nuestro; para lograr esto es necesario ajustar las variables epidemiológicas implementadas en los servicios de atención, a las establecidas internacionalmente. La implementación de estas medidas permitiría resolver dos problemas: A corto plazo, identificar las causas de desafiliación laboral entre los trabajadores que presentan TRM y a mediano o largo plazo, crear políticas encaminadas a generar estrategias de prevención y rehabilitación temprana.

\section{AGRADECIMIENTOS}

Al Departamento de Estadística del Hospital Universitario La Samaritana.

\section{REFERENCIAS}

1. Cripps RA, Lee BB, Wing P, Weerts E, Mackay J, Brown D. A global map for traumatic spinal cord injury epidemiology: towards a living data repository for injury prevention. Spinal Cord 2011 Apr;49(4):493-501. 
2. Burney RE, Maio RF, Maynard F, Karunas R. Incidence, characteristics, and outcome of spinal cord injury at trauma centers in North America. Arch Surg 1993 May;128(5): 596-599.

3. Collie A, Keating C, Pezzullo L, Gabbe B, Cooper J, Brown D, et al. Brain and spinal cord injury in Australia - economic cost and burden of disease. Inj Prev 2010;16:A25-A26

4. Mahabaleshwarkar R, Khanna R. National hospitalization burden associated with spinal cord injuries in the United States. Spinal Cord 2014 Feb;52(2):139-144.

5. Cao Y, Chen Y, DeVivo MJ. Lifetime Direct Costs After Spinal Cord Injury. Top Spinal Cord Inj Rehabil 2011;16(4):10-16.

6. Fitzharris M, Cripps RA, Lee BB. Estimating the global incidence of traumatic spinal cord injury. Spinal Cord 2014 Feb;52(2):117-122.

7. Rahimi-Movaghar V, Sayyah MK, Akbari H, Khorramirouz R, Rasouli MR, et al. Epidemiology of traumatic spinal cord injury in developing countries: a systematic review. Neuroepidemiology 2013;41(2):65-85.

8. Carvajal C, Pacheco C, Gómez C, Calderón J, Cadavid C, Jaimes F. Características clínicas y demográficas de pacientes con trauma raquimedular. Experiencia de seis años. Acta Médica Colombiana[Revista en internet]. 2015;40(1):45-50. Disponible en: http://www.redalyc.org/articulo.oa?id= 163138615009.

9. von Elm E1, Altman DG, Egger M, Pocock SJ, Gøtzsche PC, Vandenbroucke JP; STROBE Initiative. The Strengthening the Reporting of Observational Studies in Epidemiology (STROBE) statement: guidelines for reporting observational studies. Ann Intern Med 2007 Oct 16;147(8):573-577.

10. Schag CC, Heinrich RL, Ganz PA. Karnofsky performance status revisited: reliability, validity, and guidelines. J Clin Oncol 1984 Mar;2(3):187-193.

11. Charlson ME, Pompei P, Ales KL, MacKenzie CR. A new method of classifying prognostic comorbidity in longitudinal studies: development and validation. J Chronic Dis 1987;40(5):373-383.

12. Azimi P,Mohammadi HR, Azhari S, Alizadeh P, Montazeri A. AO Spine classification and Injury Severity System for Traumatic Fractures of the Spine Asian J Neurosurg 2015 Oct-Dec;10(4):282-285.

13. Cohen ME, Ditunno JF Jr, Donovan WH, Maynard FM Jr. A test of the 1992 International Standards for Neurological and
Functional Classification of Spinal Cord Injury. Spinal Cord 1998 Aug;36(8):554-560.

14. Garzón ME. Trauma raquimedular. Factores predictivos de recuperación neurológica a largo plazo. Repertorio de Cirugía y Medicina[Revista en internet]. 2005;14(2):74-78. Disponible en: http://repertorio.fucsalud.edu.co/index.php/ volumenes/revista-repertorio-de-medicina-y-cirugia-2005/ volumen-14-no-2/353-trauma-raquimedular-factores-predictivos-de-recuperacion-neurologica-a-largo-plazo.html.

15. Bajracharya S, Singh M, Singh GK, Shrestha BP. Clinicoepidemiological study of spinal injuries in a predominantly rural population of eastern Nepal: A 10 years' analysis. Indian J Orthop 2007 Oct;41(4):286-289.

16. Karamehmetoğlu SS, Nas K, Karacan I, Sarac AJ, Koyuncu H, Ataoğlu S, et al. Traumatic spinal cord injuries in southeast Turkey: an epidemiological study Spinal Cord 1997 Aug;35(8):531-533.

17. Majdan M, Brazinova A, Mauritz W. Epidemiology of traumatic spinal cord injuries in Austria 2002-2012. Eur Spine J 2016;25(1):62-73.

18. Jain NB, Ayers GD, Peterson EN, Harris MB, Morse L, O'Connor KC, et al. Traumatic spinal cord injury in the United States, 1993-2012. JAMA 2015 Jun 9;313(22):2236-2243.

19. Singh A, Tetreault L, Kalsi-Ryan S, Nouri A, Fehlings MG. Global prevalence and incidence of traumatic spinal cord injury. Clin Epidemiol 2014 Sep 23;6:309-331.

20. Liu P, Yao Y, Liu MY, Fan WL, Chao R, Wang ZG, et al. Spinal trauma in mainland China from 2001 to 2007: an epidemiological study based on a nationwide database. Spine (Phila Pa 1976). 2012 Jul 1;37(15):1310-1315.

21. Knútsdóttir S, Thórisdóttir H, Sigvaldason K, Jónsson H Jr, Björnsson A, Ingvarsson P. Epidemiology of traumatic spinal cord injuries in Iceland from 1975 to 2009. Spinal Cord 2012 Feb;50(2):123-126.

22. Li J, Liu G, Zheng Y, Hao C, Zhang Y, Wei B. The epidemiological survey of acute traumatic spinal cord injury (ATSCI) of 2002 in Beijing municipality. Spinal Cord 2011 Jul;49(7): 777-782.

23. van Middendorp JJ, Hosman AJ, Pouw MH; EM-SCI Study Group, Van de Meent H. ASIA impairment scale conversion in traumatic SCI: is it related with the ability to walk? A descriptive comparison with functional ambulation outcome measures in 273 patients. Spinal Cord 2009 Jul;47(7):555-560. 